\title{
REALCOMPACTIFICATIONS OF PRODUCTS OF ORDERED SPACES
}

\author{
WILLIAM G. MCARTHUR
}

Abstract. The equality $v(X \times Y)=v X \times v Y$ is studied for the case when one of the factors is a linearly ordered topological space (LOTS). Among the results obtained are the following:

1. If $X$ is any separable realcompact space and $Y$ is any LOTS of nonmeasurable cardinal, then $v(X \times Y)=v X \times v Y$.

2. If $X$ is a nonparacompact LOTS, then there is a paracompact LOTS $Y$ such that $v(X \times Y) \neq v X \times v Y$.

3. For any pair $X, Y$ of well-ordered spaces, $v(X \times Y)=$ $v X \times v Y$.

The circumstance in which the Hewitt realcompactification $v(X \times Y)$ of the product $X \times Y$ and the product $v X \times v Y$ of the respective Hewitt realcompactifications of the factors are equivalent extensions of the space $X \times Y$ has been studied by many researchers in a variety of contexts. The question of the equivalence of these two extensions was first considered by Hewitt in his fundamental paper [11]. Since then, the problem has been attacked from the points of view of general topology ([1], [2], [3], [8], [9], $[17])$, uniform space theory $([\mathbf{1 0}],[12])$, function space theory $([\mathbf{1 3}],[14]$, [15]), and hybrid theories [16]. A complete understanding of the properties of the spaces $X, Y$, and $X \times Y$ that essentially influence the equality $v(X \times Y)=v X \times v Y$ has thus far proved to be elusive. The rather esoteric notion of a measurable cardinal [7, p. 161] appears with annoying regularity in the theory. As we indicate in $\$ 2$ below, cardinalities of the spaces in question can be essential to the validity of the equality $v(X \times Y)=v X \times v Y$ apart from the measurability pathology. Spaces in which the continuous real-valued functions are completely determined by the compact subsets ( $k^{\prime}$-spaces) seem to play an important role ([2], [13], [15]). We suspect that the $k^{\prime}$-spaces are relevant to the problem simply because these are the spaces which have nicely behaving real-valued function spaces equipped with the compact-open topology. In fact, one can find many of the known theorems for $v(X \times Y)=v X \times v Y$ implicit in the chapter on function spaces in $[4]$.

Presented to the Society, March 9, 1972; received by the editors June 15, 1972.

AMS (MOS) subject classifications (1970). Primary 54D60, 54F05; Secondary 54B10, 54C45.

Key words and phrases. Hewitt realcompactification, ordered space, product space, realcompact space.

(c) American Mathematical Society 1973 
In the present paper, we retreat from the general context to consider the equality $v(X \times Y)=v X \times v Y$ in the more restrictive situation when one of the spaces $X$ or $Y$ is a linearly ordered topological space (hereinafter denoted as "LOTS"). Among the results obtained is the fact that in the category of well-ordered topological spaces (such a space will hereinafter be denoted as "WOTS") $v(X \times Y)=v X \times v Y$ holds universally with no restriction on cardinality. Further, it is established that if $X$ is a separable realcompact space ("space" will always mean completely regular Hausdorff space in the sequel) and $Y$ is any LOTS of nonmeasurable cardinal, then $v(X \times Y)=v X \times v Y$. It is finally determined that if $X$ is a nonparacompact LOTS, then there is a paracompact LOTS $Y$ such that card $X=$ card $Y$ and $v(X \times Y) \neq v X \times v Y$.

1. Terminology and notation. Our basic references for notation are [6] and [7]. For the convenience of the reader, we summarize below the pertinent parts of [6] and other definitions and results which will be referred to in the sequel.

1.1. For a space $X, v X$ denotes the Hewitt realcompactification of $X$ [7, pp. 116-119]. The symbolism $v(X \times Y)=v X \times v Y$ means that the space $X \times Y$ is $C$-embedded in the space $v X \times v Y$.

1.2. For an ordinal $\sigma, W(\sigma)$ denotes the ordinal space of all ordinals less than $\sigma$ equipped with the order topology.

1.3. The smallest ordinal of cardinality $\boldsymbol{\aleph}_{\alpha}$ is denoted by $\omega_{\alpha}$; we will take the point of view that the only distinction between $\boldsymbol{\aleph}_{\alpha}$ and $\omega_{\alpha}$ is one of notation.

1.4. Let $L$ be a LOTS. A gap of $L$ is defined as a Dedekind cut $(A \mid B)$ such that $A$ has no last element and $B$ no first. If $A=\varnothing$ or $B=\varnothing,(A \mid B)$ is called an end-gap. The linearly ordered set consisting of $L$ together with all gaps of $L$ is denoted by $L^{+}$.

1.5. Let $L$ be a LOTS and let $u=(A \mid B)$ be a gap of $L$. If $u$ is not a left end-gap, then the left character of $u$ is the least ordinal $\lambda$ which is cofinal with $A$. If $u$ is not a right end-gap, then the right character of $u$ is the least ordinal $\rho$ such that $\rho^{*}$ (“*” denotes reverse order) is coinitial with $B$.

1.6. (i) Let $L$ be a LOTS and let $\omega_{\alpha}$ be any regular initial ordinal. An increasing or decreasing sequence $s=\left\{x_{\sigma}\right\}_{\sigma<\omega_{\alpha}}$ of points of $L^{+}$is a $Q$ sequence if for every nonzero limit ordinal $\lambda<\omega_{\alpha}$, the limit in $L^{+}$of the segment $\left\{x_{\sigma}\right\}_{\sigma<\lambda}$ of $s$ is a gap of $L$.

(ii) If $s$ is a $Q$-sequence and if the gap $u$ is the limit of the entire sequence $s, s$ is called a $Q$-sequence at $u$.

(iii) A gap $u$ is called a $Q$-gap from the left (right) if there exists an increasing (decreasing) $Q$-sequence at $u$.

(iv) A gap $u$ is called a $Q$-gap if it is a $Q$-gap from the left and from the right (or the appropriate one if $u$ is an end-gap). 
1.7. Let $X$ be a LOTS. For each gap $u$ of $X$ add elements $l_{u}$ and $r_{u}$ (or only the appropriate one if $u$ is an end-gap) to form the LOTS $X^{\prime \prime}$ ordered in the natural way $\left(l_{u}<r_{u}\right)$ preserving the order of $X$. Form $X^{\prime}$ from $X^{\prime \prime}$ by deleting every element $l_{u}$ for which the gap $u$ is a $Q$-gap from the left, and every $r_{u}$ for which $u$ is a $Q$-gap from the right. Then, $X$ is a dense $C$-embedded subspace of $X^{\prime}$ and hence $X \subset X^{\prime} \subset v X$. If card $X$ is nonmeasurable, then $X^{\prime}=v X$ (see [6, pp. 359-360]).

1.8. (Gilman and Henriksen). A LOTS $X$ is paracompact if and only if every gap of $X$ is a $Q$-gap. A LOTS $X$ is realcompact if and only if every gap of $X$ is a nonmeasurable $Q$-gap.

1.9. (Glicksberg). If $X \times Y$ is pseudocompact, then $v(X \times Y)=v X \times v Y$.

1.10. (Comfort). If $X$ is locally compact, realcompact of nonmeasurable cardinal, then $v(X \times Y)=v X \times v Y$ for every space $Y$.

1.11. (Hager). If $X$ and $Y$ are spaces such that $X=\bigcup_{n} X_{n}, Y=\bigcup_{n} Y_{n}$, $X_{n}$ is completely separated from $X-X_{n+1}$ and $Y_{n}$ is completely separated from $Y-Y_{n+1}$ for each $n$, and $X_{n} \times Y_{n}$ is pseudocompact for each $n$, then $v(X \times Y)=v X \times v Y$.

2. Linearly ordered spaces and $v(X \times Y)$. It is established in [6] that a gap $u$ of a LOTS $X$ is not a $Q$-gap from the left (right) if and only if every real-valued continuous function on $X$ is constant on a left (right) interval at $u$. The following theorem indicates that the "size" of the factors can in some cases determine the validity of the equality $v(X \times Y)=v X \times v Y$.

THEOREM 2.1. Suppose $X$ is a nonparacompact LOTS of nonmeasurable cardinal. Let $\left\{u_{\lambda}\right\}_{\lambda \in \Lambda}$ be the family of all gaps of $X$ which are not $Q$-gaps from the left and $\left\{u_{\rho}\right\}_{\rho \in P}$, be the family of all gaps of $X$ which are not $Q$-gaps from the right. Let $\left\{\omega_{\alpha}\right\}_{\alpha \in A}$ be the collection of left characters of $\left\{u_{\lambda}\right\}_{\lambda \in \Lambda}$ and $\left\{\omega_{\beta}\right\}_{\beta \in B}$ be the collection of right characters of $\left\{u_{\rho}\right\}_{\rho \in P}$. Let $\omega_{\gamma}=\inf \left\{\omega_{\xi}\right.$ : $\xi \in A \cup B\}$. Let $Y$ be a realcompact space which has a dense subspace $D$ such that card $D<\omega_{\gamma}$. Then $v(Y \times X)=v Y \times v X$.

Proof. Let $f$ be a continuous real-valued function on $Y \times X$. We wish to extend $f$ continuously to $Y \times X^{\prime}$. For each point $y$ of $Y,\left.f\right|_{\{y\} \times X^{\prime}}$ can be extended continuously to the function $\hat{f}_{y}$ from $\{y\} \times X^{\prime}$ to the real line. Define $\hat{f}(y, p)=\hat{f}_{y}(p)$ for $y$ in $Y$ and $p$ in $X^{\prime}$. To show that $\hat{f}$ is the desired extension of $f$, it suffices to show that, for each point $p$ of $X^{\prime}-X, \hat{f}$ is continuous on the space $Y \times(X \cup\{p\})$. Let $p$ be a point of $X^{\prime}-X$. We will assume that $p=l_{u_{\lambda}}$ for some $\lambda \in \Lambda$, the other case being similar. For each point $d$ of $D$, there is a real number $\eta_{d}$ and a point $x_{d}$ of $X$ such that $\hat{f}_{d}$ is constantly equal to $\eta_{d}$ on $\{d\} \times\left[x_{d}, l_{u_{\lambda}}\right]$ (see $[6,10.6]$ ). Our cardinality hypothesis on the set $D$ ensures that we may choose a point $x^{\prime}$ of $X$ such that $x_{d}<x^{\prime}<l_{u_{\lambda}}$ for every $d$ in $D$. It is easy to see that, for every point $y$ of 
$Y, \hat{f}_{y}$ is constant on $\{y\} \times\left[x^{\prime}, l_{u_{\lambda}}\right]$. Now, choose any point $x^{\prime \prime}$ of $X$ such that $x^{\prime}<x^{\prime \prime}<l_{u_{\lambda}}$. Let $y_{0}$ be any point of $Y$ and $\varepsilon>0$. Then, there is a neighborhood $U$ of $y_{0}$ and a neighborhood $\left(x_{1}, x_{2}\right)$ of $x^{\prime \prime}$ with $x^{\prime} \leqq x_{1}<x^{\prime \prime}<x_{2}<l_{u_{\lambda}}$ such that $f_{*}\left(U \times\left(x_{1}, x_{2}\right)\right) \subset\left(f\left(y_{0}, x^{\prime \prime}\right)-\varepsilon, \quad f\left(y_{0}, x^{\prime \prime}\right)+\varepsilon\right)$. Then, $\hat{f}_{*}\left(U \times\left(x_{1}, l_{u_{\lambda}}\right]\right) \subset\left(f\left(y_{0}, x^{\prime \prime}\right)-\varepsilon, f\left(y_{0}, x^{\prime \prime}\right)+\varepsilon\right)=\left(\hat{f}\left(y_{0}, l_{u_{\lambda}}\right)-\varepsilon, \hat{f}\left(y_{0}, l_{u_{\lambda}}\right)+\varepsilon\right)$. [The fact that $\left(x_{1}, l_{u_{\lambda}}\right]$ is a neighborhood of $l_{u_{\lambda}}$ in $X \cup\left\{l_{u_{\lambda}}\right\}$ is evident from the construction of $X^{\prime \prime}$, noting that $\left.\left(x_{1}, l_{u_{\lambda}}\right]=\left(x_{1}, r_{u_{\lambda}}\right) \cap\left(X \cup\left\{l_{u_{\lambda}}\right\}\right).\right] ! !$

COROLlary 2.2. Let $Y$ be a separable realcompact space. Then, for every LOTS $X$ of nonmeasurable cardinal, $v(X \times Y)=v X \times v Y$.

Proof. If $X$ is paracompact, then $X$ is realcompact and the situation is trivial. If $X$ is nonparacompact, then the result follows from 2.1 with the remark that $\omega_{1} \leqq \omega_{\gamma}$ since a gap with left (right) character $\omega_{0}$ is a $Q$-gap from the left (right).!!

Corollary 2.3. Let Y be a countable space. Then, for every LOTS $Y$ of nonmeasurable cardinal, $v(X \times Y)=v X \times v Y$.

RemarK 2.4. It was established in [16] that there are spaces $X$ and $Y$ with $X$ countable and $Y$ with nonmeasurable cardinal such that $v(X \times Y) \neq$ $v X \times v Y$. In fact, it can be shown that if $Y$ is any nonrealcompact extension of the countable discrete space, then there is a countable space $X$ such that $v(X \times Y) \neq v X \times v Y$.

THEOREM 2.5. If $X$ is a nonparacompact LOTS, then there is a paracompact LOTS $Y$ such that $v(X \times Y) \neq v X \times v Y$.

Proof. Let $u$ be a gap of $X$ which is not a $Q$-gap. We assume for definiteness that $u$ is not a $Q$-gap from the left. Let $\left\{x_{\sigma}\right\}_{\sigma<\omega_{\alpha}}$ be an increasing sequence in $X$ with limit $u$. Let $E=\left\{x_{\sigma}: \sigma\right.$ is a nonlimit ordinal $\left.<\omega_{\alpha}\right\}$ and let $F=E \times Z$ (" $Z$ " denotes the discrete space of integers) be equipped with the lexicographic order. Finally, let $Y=F \cup\left\{l_{u}\right\}$ be equipped with the order topology where $l_{u}$ follows all elements of $F$. Since $F$ is a discrete subspace of $Y, Y$ is paracompact. For each $x_{\sigma}$ in $E$, let $f_{\sigma}$ be a continuous function from $X \cup\left\{l_{u}\right\}$ to the closed interval $[0,1]$ such that $f_{\sigma}\left(l_{u}\right)=0$ and $f_{\sigma}(x)=1$ for every $x \leqq x_{\sigma}$ and every $x>l_{u}$. [We can do this since $\{x$ : $\left.x \leqq x_{\sigma}\right\} \cup\left\{x: x>l_{u}\right\}$ is closed.] Define the real-valued function $f$ on $X \times Y$ by the rule:

$$
\begin{aligned}
f(x, y) & =1, & & \text { if } y=l_{u}, \\
& =f_{\sigma}(x), & & \text { if } y=\left(x_{\sigma}, z\right) .
\end{aligned}
$$

It is not hard to see that $f$ is continuous on $X \times Y$. We show that $f$ cannot be continuously extended to the point $\left(l_{u}, l_{u}\right)$ of $v X \times v Y$. Let $U$ be a neighborhood of $\left(l_{u}, l_{u}\right)$ in $v X \times v Y$. Choose a point $x_{\sigma}$ of $E$ such that 
$(x, y)$ is in $U$ whenever $x_{\sigma}<x<l_{u}$ and $\left(x_{\sigma}, 0\right)<y$. Choose a point $x$ of $X$ such that $x_{\sigma}<x<l_{u}$ and $f_{\sigma}(x)<\frac{1}{2}$. Then, $\left(x, l_{u}\right)$ and $\left(x,\left(x_{\sigma}, 1\right)\right)$ are both points of $U$ and $\left|f\left(x, 1_{u}\right)-f\left(x,\left(x_{\sigma}, 1\right)\right)\right|=\left|1-f_{\sigma}(x)\right|>\frac{1}{2}$. Thus, $f$ cannot be continuously extended over $v X \times v Y$.!!

REMARK 2.6. If the LOTS $X$ in 2.5 has nonmeasurable cardinal, then $Y$ is realcompact. We may construct a space $T$ from $Y$ by an appropriate topological sum so that $T$ is a paracompact LOTS, card $T=$ card $X$, and $v(X \times T) \neq v X \times v T$. Thus, barring measurable cardinals, we have shown that if $X$ is a nonrealcompact LOTS, there is a realcompact LOTS $T$ such that card $T=\operatorname{card} X$ and $v(X \times T) \neq v X \times v T$.

REMARK 2.7. Theorem 2.5 also indicates that the cardinality criterion of Theorem 2.1 is critical. Notice that the cardinality of $Y$ in 2.5 is precisely the left character of the gap $u$.

3. Well ordered spaces and $v(X \times Y)$. The situation for well-ordered spaces is simple and satisfactory. Our main result of this section is that, with no restriction on cardinality, $v(X \times Y)=v X \times v Y$ always holds in the category of well-ordered topological spaces. We begin with a few preliminary results.

\section{LEMMA 3.1. Every paracompact WOTS is realcompact.}

Proof. If $W(\sigma)$ is a paracompact, noncompact WOTS, then $\sigma$ is an endgap of character $\omega_{0}$. Thus, $W(\sigma)$ is a countable union of compact subspaces and is realcompact $[7,8.2] . !$ !

LEMMA 3.2. A nonparacompact WOTS is pseudocompact.

Proof. Let $W(\sigma)$ be a nonparacompact WOTS. Suppose $f$ is a realvalued continuous function defined on $W(\sigma)$. Then, there is an ordinal $\tau<\sigma$ such that $f$ is constant on the interval $[\tau, \sigma)$. But, the interval $[0, \tau]$ is compact and hence $f$ is bounded on the set $[0, \tau] \cup[\tau, \sigma)=W(\sigma)$.!!

LEMMA 3.3. If $W(\sigma)$ and $W(\tau)$ are pseudocompact WOTS, then $W(\sigma) \times$ $W(\tau)$ is pseudocompact.

Proof. Note that every WOTS is locally compact. The result now follows from [5, 3.3 and 3.4].!!

We now state and prove the main result of the section.

THEOREM 3.4. For each pair of ordinals $\sigma$ and $\tau, v(W(\sigma) \times W(\tau))=$ $v(W(\sigma)) \times v(W(\tau))$.

Proof. Case I. Both $W(\sigma)$ and $W(\tau)$ are realcompact.

There is nothing to prove in this case.

Case II. Both $W(\sigma)$ and $W(\tau)$ are nonrealcompact. 
By 3.1, 3.2, and 3.3, we conclude that $W(\sigma) \times W(\tau)$ is pseudocompact. But, then by Glicksberg's theorem (see 1.9), $v(W(\sigma) \times W(\tau))=v(W(\sigma)) \times$ $v(W(\tau))$.

Case III. $W(\sigma)$ is realcompact and $W(\tau)$ is nonrealcompact.

If $W(\sigma)$ is compact, then Glicksberg's theorem implies the result. If $W(\sigma)$ is noncompact, choose an increasing sequence $x_{1}, x_{2}, \cdots$ of nonlimit ordinals whose limit is $\sigma$. Then, $W(\sigma)=\bigcup_{n=1}^{\infty} W\left(x_{n}\right)$, each $W\left(x_{n}\right)$ is completely separated from $W(\sigma)-W\left(x_{n+1}\right)$, and each $W\left(x_{n}\right) \times W(\tau)$ is pseudocompact ( $W\left(x_{n}\right)$ is compact and $W(\tau)$ is pseudocompact). Thus, by Hager's theorem (see 1.11), $v(W(\sigma) \times W(\tau))=v(W(\sigma)) \times v(W(\tau))$.!!

COROLlaRY 3.5. For every pair of WOTS $X$ and $Y, v(X \times Y)=v X \times v Y$.

Proof. There are unique ordinals $\sigma$ and $\tau$ such that $X$ is homeomorphic to $W(\sigma)$ and $Y$ is homeomorphic to $W(\tau)$.!!

REMARK 3.6. Recall that, as a consequence of Theorem 2.5, for every nonrealcompact WOTS $X$, there is a paracompact LOTS $Y$ such that $v(X \times Y) \neq v X \times v Y$. If $X$ is a realcompact WOTS with nonmeasurable cardinal, then $v(X \times Y)=v X \times v Y$ for every space $Y$ by Comfort's theorem (see 1.10).

\section{REFERENCES}

1. W. W. Comfort, Locally compact realcompactifications, General Topology and Its Relations to Modern Analysis and Algebra (Proc. Second Prague Topological Sympos., 1966), II, Academic Press, New York, 1967, pp. 95-100.

2. - On the Hewitt realcompactification of a product space, Trans. Amer. Math. Soc. 131 (1968), 107-118. MR 36 \#5896.

3. W. W. Comfort and S. Negrepontis, Extending continuous functions on $X \times Y$ to subsets of $\beta X \times \beta Y$, Fund. Math. 59 (1966), 1-12. MR 34 \#782.

4. J. Dugundji, Topology, Allyn and Bacon, Boston, Mass., 1966. MR 33 \#1824.

5. Z. Frolik, The topological product of two pseudo-compact spaces, Czechoslovak Math. J. 10 (95) (1960), 339-349. MR 22 \#7099.

6. L. Gillman and M. Henriksen, Concerning rings of continuous functions, Trans. Amer. Math. Soc. 77 (1954), 340-362. MR 16, 156.

7. L. Gillman and M. Jerison, Rings of continuous functions, University Series in Higher Math., Van Nostrand, Princeton, N.J., 1960. MR 22 \#6994.

8. I. Glicksberg, Stone-Čech compactifications of products, Trans. Amer. Math. Soc. 90 (1959), 369-382. MR 21 \#4405.

9. A. W. Hager, On the tensor product of function rings, Doctoral Dissertation, Pennsylvania State Univ., University Park, Pa., 1965.

10. - Projections of zero-sets (and the fine uniformity on a product), Trans. Amer. Math. Soc. 140 (1969), 87-94. MR 39 \#3448.

11. E. Hewitt, Rings of real-valued continuous functions. I, Trans. Amer. Math. Soc. 64 (1948), 45-99. MR 10, 126.

12. J. R. Isbell, Uniform spaces, Math. Surveys, no. 12, Amer. Math. Soc., Providence, R.I., 1964. MR 30 \#561. 
13. M. Husek, The Hewitt realcompactification of a product, Comment. Math. Univ. Carolinae 11 (1970), 393-395. MR 42 \#2438.

14. —- Pseudo-m-compactness and $v(P \times Q)$ (to appear).

15. - Realcompactness of function spaces and $v(P \times Q)$ (to appear).

16. W. McArthur, Hewitt realcompactifications of products, Canad. J. Math. 22 (1970), 645-656. MR 42 \#1069.

17. —_ A note on homeomorphic realcompactifications, Arch. Math. 22 (1971), 85-87.

Department of Mathematics, Shippensburg State College, Shippensburg, Pennsylvania 17257 